\title{
ON ISOLATED POINTS OF THE SPECTRUM OF A BOUNDED LINEAR OPERATOR
}

\author{
CHRISTOPH SCHMOEGER
}

(Communicated by Palle E. T. Jorgensen)

\begin{abstract}
For a bounded linear operator $A$ on a Banach space we characterize the isolated points in the spectrum of $A$, the Riesz points of $A$, and the poles of the resolvent of $A$.
\end{abstract}

\section{TERMINOLOGY AND INTRODUCTION}

Throughout this paper $E$ will be an infinite-dimensional complex Banach space and $A$ will be a bounded linear operator on $E$. We denote by $N(A)$ the kernel and by $A(E)$ the range of $A$. The spectrum of $A$ will be denoted by $\sigma(A)$. The resolvent set $\varrho(A)$ of $A$ is the complement of $\sigma(A)$ in the complex plane $\mathbb{C}$. For any $\lambda$ in $\varrho(A)$ the resolvent operator $(\lambda I-A)^{-1}$ is denoted by $R_{\lambda}(A)$.

Let $\lambda_{0}$ be an isolated point in $\sigma(A)$. The spectral projection corresponding to $\lambda_{0}$ will be denoted by $P_{\lambda_{0}}$. We have $E=P_{\lambda_{0}}(E) \oplus N\left(P_{\lambda_{0}}\right)$.

In [3] Mbekhta introduced two important subspaces of $E$ :

$$
\begin{gathered}
K(A)=\left\{x \in E: \begin{array}{l}
\text { there exist } c>0 \text { and a sequence }\left(x_{n}\right)_{n \geq 1} \subseteq E \\
\text { such that } A x_{1}=x, \quad A x_{n+1}=x_{n} \text { for all } n \in \mathbb{N}, \\
\left.\quad \text { and }\left\|x_{n}\right\| \leq c^{n}\|x\| \text { for all } n \in \mathbb{N}\right\}, \\
H_{0}(A)=\left\{x \in E: \lim _{n \rightarrow \infty}\left\|A^{n} x\right\|^{1 / n}=0\right\}
\end{array}\right.
\end{gathered}
$$

and proved the following

Theorem 1. $A$ point $\lambda_{0} \in \sigma(A)$ is isolated in $\sigma(A)$ if and only if there is a bounded projection $P$ on $E$ such that

$$
P(E)=H_{0}\left(\lambda_{0} I-A\right) \text { and } N(P)=K\left(\lambda_{0} I-A\right) .
$$

In the present paper we shall prove that $\lambda_{0} \in \sigma(A)$ is an isolated point of $\sigma(A)$ if and only if $K\left(\lambda_{0} I-A\right)$ is closed and $E=K\left(\lambda_{0} I-A\right) \oplus H_{0}\left(\lambda_{0} I-A\right)$ (where $\oplus$ denotes the algebraically direct sum). This characterization leads to

Received by the editors March 4, 1991 and, in revised form, June 24, 1991.

1991 Mathematics Subject Classification. Primary 47A10; Secondary 47B06.

Key words and phrases. Local spectral theory, isolated points of the spectrum, Riesz points. 
a characterization of the poles of the resolvent of $A$ and to a characterization of the Riesz points of $A$. This will be done in $\S 3$ of this paper.

\section{Preliminary Results}

The operator $A$ is said to have the single-valued extension property (SVEP) in $\lambda_{0} \in \mathbb{C}$ if for any holomorphic function $f: U \rightarrow E$, where $U$ is a neighbourhood of $\lambda_{0}$, with $(\lambda I-A) f(\lambda) \equiv 0$, the result is $f(\lambda) \equiv 0$. We say that $A$ has the SVEP if $A$ has the SVEP in each $\lambda \in \mathbb{C}$.

The following theorem collects some results due to Mbekhta (see [4]).

Theorem 2. (a) $A(K(A))=K(A)$ and $A\left(H_{0}(A)\right) \subseteq H_{0}(A)$;

(b) $A$ has the SVEP in $\lambda_{0}$ if $H_{0}\left(\lambda_{0} I-A\right)$ is closed;

(c) $A$ has the SVEP in $\lambda_{0}$ if and only if $K\left(\lambda_{0} I-A\right) \cap H_{0}\left(\lambda_{0} I-A\right)=\{0\}$.

The proof of the next result is immediate.

Proposition 1. Let $x \in H_{0}(A)$ and define the function $g$ on $\mathbb{C} \backslash\{0\}$ by

$$
g(\lambda)=\sum_{n=0}^{\infty} \frac{A^{n} x}{\lambda^{n+1}} .
$$

Then $g$ is holomorphic and $(\lambda I-A) g(\lambda)=x$ for all $\lambda \in \mathbb{C} \backslash\{0\}$.

Proposition 2. Let $F$ be a closed subspace of $E$ such that $A(F)=F$. Then $F \subseteq K(A)$.

Proof. Since $F$ is a Banach space and $A(F)=F$, the open mapping theorem shows the existence of a constant $c>0$ so that

$$
\text { for each } u \in F \text { there exists } v \in F \text { such that }
$$

$$
A v=u \text { and }\|v\| \leq c\|u\| \text {. }
$$

Let $x \in F$. Use (2.1) to construct a sequence $\left(x_{n}\right)_{n \geq 1} \subseteq F$ such that $A x_{1}=x$, $A x_{n+1}=x_{n}$, and $\left\|x_{n}\right\| \leq c^{n}\|x\|$. It follows that $x \in K(A)$.

Let us review the classical definitions of ascent and descent. The ascent $p(A)$ and the descent $q(A)$ are the extended integers given by

$$
\begin{aligned}
& p(A)=\inf \left\{n \geq 0: N\left(A^{n}\right)=N\left(A^{n+1}\right)\right\}, \\
& q(A)=\inf \left\{n \geq 0: A^{n}(E)=A^{n+1}(E)\right\} .
\end{aligned}
$$

The infimum over the empty set is taken to be $\infty$. It follows from [2, Satz 72.3] that if $p(A)$ and $q(A)$ are both finite then they are equal.

We have the following characterization of the poles of the resolvent of $A$ (see [2, Satz 101.2]):

Theorem 3. The complex number $\lambda_{0}$ is a pole of $R_{\lambda}(A)$ if and only if $0<$ $p\left(\lambda_{0} I-A\right)=q\left(\lambda_{0} I-A\right)<\infty$. In this case we have

$$
P_{\lambda_{0}}(E)=N\left(\left(\lambda_{0} I-A\right)^{p}\right) \text { and } N\left(P_{\lambda_{0}}\right)=\left(\lambda_{0} I-A\right)^{p}(E) \text {, }
$$

where $p=p\left(\lambda_{0} I-A\right)$ is the order of the pole $\lambda_{0}$.

The next proposition is a generalization of [1, Theorem 2]. 
Proposition 3. Suppose that $A$ has the SVEP in $\lambda_{0}=0$ and $q(A)<\infty$. Then $p(A)=q(A)$.

Proof. Let $q=q(A), B=A^{q}$, and $\widehat{E}=E / N(B)$. Since $N(B)$ is closed, $\widehat{E}$ is a Banach space. Let $\widehat{B}: \widehat{E} \rightarrow E$ be the corresponding canonical injection. It is easy to see that the operator $\widehat{B}^{-1}: A^{q}(E) \rightarrow \widehat{E}$ is closed, thus $A^{q}(E)$ is the domain of a closed linear operator. Since $A\left(A^{q}(E)\right)=A^{q}(E)$ and $A$ has the SVEP in $0,\left[1\right.$, Corollary 4] shows that $N(A) \cap A^{q}(E)=\{0\}$. Use [2, Satz 72.1] to derive $p(A)<\infty$.

Corollary 1. The following assertions are equivalent:

(a) 0 is a pole of $R_{\lambda}(A)$;

(b) $A$ has the SVEP in 0 and $q(A)<\infty$.

Proof. (a) implies (b). Since 0 is isolated in $\sigma(A), A$ has the SVEP in 0 . Theorem 3 shows that $q(A)<\infty$.

(b) implies (a). Proposition 3 and Theorem 3.

\section{ISOLATED POINTS OF THE SPECTRUM}

The starting point of our investigation is

Proposition 4. Suppose that 0 is an isolated point in $\sigma(A)$. Then

(a) $P_{0}(E)=H_{0}(A)$;

(b) $N\left(P_{0}\right)=K(A)$.

Proof. (a) follows from [2, Satz 100.2].

(b) Since 0 is isolated in $\sigma(A), \sigma\left(A_{\mid P_{0}(E)}\right)=\{0\}$ and $0 \in \varrho\left(A_{\mid N\left(P_{0}\right)}\right)$ [2, Satz 100.1]. Then $N\left(P_{0}\right)$ is closed and $A\left(N\left(P_{0}\right)\right)=N\left(P_{0}\right)$. Hence, by Proposition 2, $N\left(P_{0}\right) \subseteq K(A)$. By Theorem 2(c), $K(A) \cap H_{0}(A)=\{0\}$. Therefore,

$$
\begin{aligned}
K(A) & =K(A) \cap E=K(A) \cap\left[N\left(P_{0}\right) \oplus P_{0}(E)\right] \\
& =N\left(P_{0}\right)+K(A) \cap H_{0}(A)=N\left(P_{0}\right) .
\end{aligned}
$$

Theorem 4. The following assertions are equivalent:

(a) 0 is an isolated point in $\sigma(A)$;

(b) $K(A)$ is closed and $E=K(A) \oplus H_{0}(A)$ ( $\oplus$ denotes the algebraically direct sum).

Proof. (a) implies (b). Use Proposition 4 or Theorem 1.

(b) implies (a). Since $K(A)$ is closed, $A(K(A))=K(A)$ (Theorem 2(a)), and $N(A) \subseteq H_{0}(A)$, the operator $A: K(A) \rightarrow K(A)$ is invertible. Hence there exists $\varepsilon>0$ such that $\lambda I-A_{\mid K(A)}$ is invertible if $|\lambda|<\varepsilon$. In particular,

$$
(\lambda I-A)(K(A))=K(A) \quad \text { if }|\lambda|<\varepsilon .
$$

Since for all $\lambda \neq 0, N(\lambda I-A) \subseteq K(A)$, we have

$$
N(\lambda I-A)=\{0\} \quad \text { if } 0<|\lambda|<\varepsilon .
$$

By Proposition 1, for all $\lambda \neq 0$,

$$
H_{0}(A) \subseteq(\lambda I-A)(E) .
$$

Now, (3.1) and (3.3) imply

$$
E=K(A) \oplus H_{0}(A) \subseteq(\lambda I-A)(E) \quad \text { if } 0<|\lambda|<\varepsilon .
$$


Consequently, $\{\lambda \in \mathbb{C}: 0<|\lambda|<\varepsilon\} \subseteq \varrho(A)$ and the proof is complete.

Now we are in a position to present the announced characterization of the poles of the resolvent of $A$.

Theorem 5. The following assertions are equivalent:

(a) 0 is a pole of the resolvent of $A$;

(b) $A$ has the SVEP in 0 and $q(A)<\infty$;

(c) There exists $p \in \mathbb{N}$ such that

$$
N\left(A^{p}\right)=H_{0}(A) \quad \text { and } \quad A^{p}(E)=K(A) ;
$$

(d) $A$ has the SVEP in 0 and there exists $p \in \mathbb{N}$ such that $K(A)=A^{p}(E)$;

(e) $q(A)<\infty$ and $H_{0}(A)$ is closed.

Proof. By Corollary 1, (a) and (b) are equivalent.

(a) implies (c). Use Theorem 3 and Proposition 4.

(c) implies (a). By Theorem 3, we have to show that $p(A)$ and $q(A)$ are both finite. Since

$$
N\left(A^{p+1}\right) \subseteq H_{0}(A)=N\left(A^{p}\right) \subseteq N\left(A^{p+1}\right),
$$

we have $p(A) \leq p$. Use Theorem $2\left(\right.$ a) to derive $A^{p+1}(E)=A\left(A^{p}(E)\right)=$ $A(K(A))=K(A)=A^{p}(E)$. Thus $q(A) \leq p$.

(a) implies (d). Use (b) and (c).

(d) implies (b). As in the proof of "(c) implies (a)," we have $A^{p}(E)=$ $A^{p+1}(E)$, hence $q(A)<\infty$.

(a) implies (e). Clear.

(e) implies (b). By Theorem 2(b), $A$ has the SVEP in 0.

The remainder of this paper deals with Riesz points and Riesz operators. A complex number $\lambda_{0}$ is called a Riesz point of $A$, if

$p\left(\lambda_{0} I-A\right)=q\left(\lambda_{0} I-A\right)<\infty$ and $\operatorname{dim} N\left(\lambda_{0} I-A\right)=\operatorname{codim}\left(\lambda_{0} I-A\right)(E)<\infty$.

Note that a Riesz point of $A$ is either a pole of the resolvent (and hence isolated in $\sigma(A))$ or a point in the resolvent set $\varrho(A)$.

Proposition 5. The complex number $\lambda_{0} \in \sigma(A)$ is a Riesz point of $A$ if and only if $\lambda_{0}$ is isolated in $\sigma(A)$ and the corresponding spectral projection is finite dimensional.

Proof. [2, Satz 105.3].

The next theorem uses the subspaces $K(A)$ and $H_{0}(A)$ and the SVEP to characterize the Riesz points of $A$.

Theorem 6. The following assertions are equivalent:

(a) 0 is a Riesz point of $A$;

(b) $K(A)$ is closed, $\operatorname{dim} H_{0}(A)<\infty$, and $E=K(A) \oplus H_{0}(A)$, where $\oplus$ denotes the algebraically direct sum;

(c) $q(A)<\infty$ and $\operatorname{dim} H_{0}(A)<\infty$;

(d) $\operatorname{dim} H_{0}(A)<\infty$ and $K(A)=A^{P}(E)$ for some $p \in \mathbb{N}$;

(e) $A$ has the SVEP in $0, q(A)<\infty$, and $\operatorname{dim} N(A)<\infty$. 
Proof. (a) $\Leftrightarrow$ (b). Proposition 4 and Theorem 4 .

(c) $\Rightarrow$ (a). Since $N\left(A^{n}\right) \subseteq N\left(A^{n+1}\right) \subseteq H_{0}(A)$ and $\operatorname{dim} H_{0}(A)<\infty$, there exists $p \in \mathbb{N}$ such that $\operatorname{dim} N\left(A^{p}\right)=\operatorname{dim} N\left(A^{p+1}\right)<\infty$. This gives $N\left(A^{p}\right)=$ $N\left(A^{p+1}\right)$, thus $p(A)<\infty$. By Theorem 3, 0 is a pole of $R_{\lambda}(A)$, hence 0 is isolated in $\sigma(A)$. Proposition 4 shows that $\operatorname{dim} P_{0}(E)<\infty$. Now use Proposition 5.

(a) $\Rightarrow$ (d). Propositions 4 and 5 show that $\operatorname{dim} P_{0}(E)=\operatorname{dim} H_{0}(A)<\infty$ and $N\left(P_{0}\right)=K(A)$. Since 0 is a pole of $R_{\lambda}(A)$, we conclude from Theorem 3 that $K(A)=A^{p}(E)$ for some $p \in \mathbb{N}$.

(d) $\Rightarrow(\mathrm{c}) \cdot A^{p+1}(E)=A(K(A))=K(A)=A^{p}(E)$, thus $q(A)<\infty$.

(a) $\Rightarrow$ (e). Clear.

(e) $\Rightarrow$ (a). By Proposition 3, $p(A)=q(A)<\infty$. [2, Satz 72.6] shows that $\operatorname{dim} N(A)=\operatorname{codim} A(E)<\infty$, thus 0 is a Riesz point of $A$.

The operator $A$ is called a Riesz operator if every $\lambda \in \sigma(A) \backslash\{0\}$ is a Riesz point of $A$.

An immediate consequence of Theorem 6 is

Theorem 7. The following assertions are equivalent:

(a) $A$ is a Riesz operator;

(b) $\operatorname{dim} H_{0}(\lambda I-A)<\infty, E=K(\lambda I-A) \oplus H_{0}(\lambda I-A)$, and $K(\lambda I-A)$ is closed for all $\lambda \in \sigma(A) \backslash\{0\}$

(c) $q(\lambda I-A)<\infty$ and $\operatorname{dim} H_{0}(\lambda I-A)<\infty$ for all $\lambda \in \sigma(A) \backslash\{0\}$;

(d) $\operatorname{dim} H_{0}(\lambda I-A)<\infty$ for all $\lambda \in \sigma(A) \backslash\{0\}$ and for each $\lambda \in \sigma(A) \backslash\{0\}$ there exists $p(\lambda) \in \mathbb{N}$ such that $K(\lambda I-A)=(\lambda I-A)^{p(\lambda)}(E)$.

\section{ACKNOWLEDGMENT}

The author wishes to thank the referee for his suggestions that led to a simplification of the proof of Theorem 4 .

\section{REFERENCES}

1. J. K. Finch, The single-valued extension property on a Banach space, Pacific J. Math. 58 (1975), 61-69.

2. H. Heuser, Funktionalanalysis, 2nd ed., Teubner, Stuttgart, 1986.

3. M. Mbekhta, Généralisation de la décomposition de Kato aux opérateurs paranormaux et spectraux, Glasgow Math. J. 29 (1987), 159-175.

4. __ Sur la théorie spectrale locale et limite des nilpotents, Proc. Amer. Math. Soc. 110 (1990), 621-631.

Mathematisches Institut 1, Universität KarlsRuhe, ENGlerstr. 2, D-7500 KarlsRuhe 1 , GeRMANY 